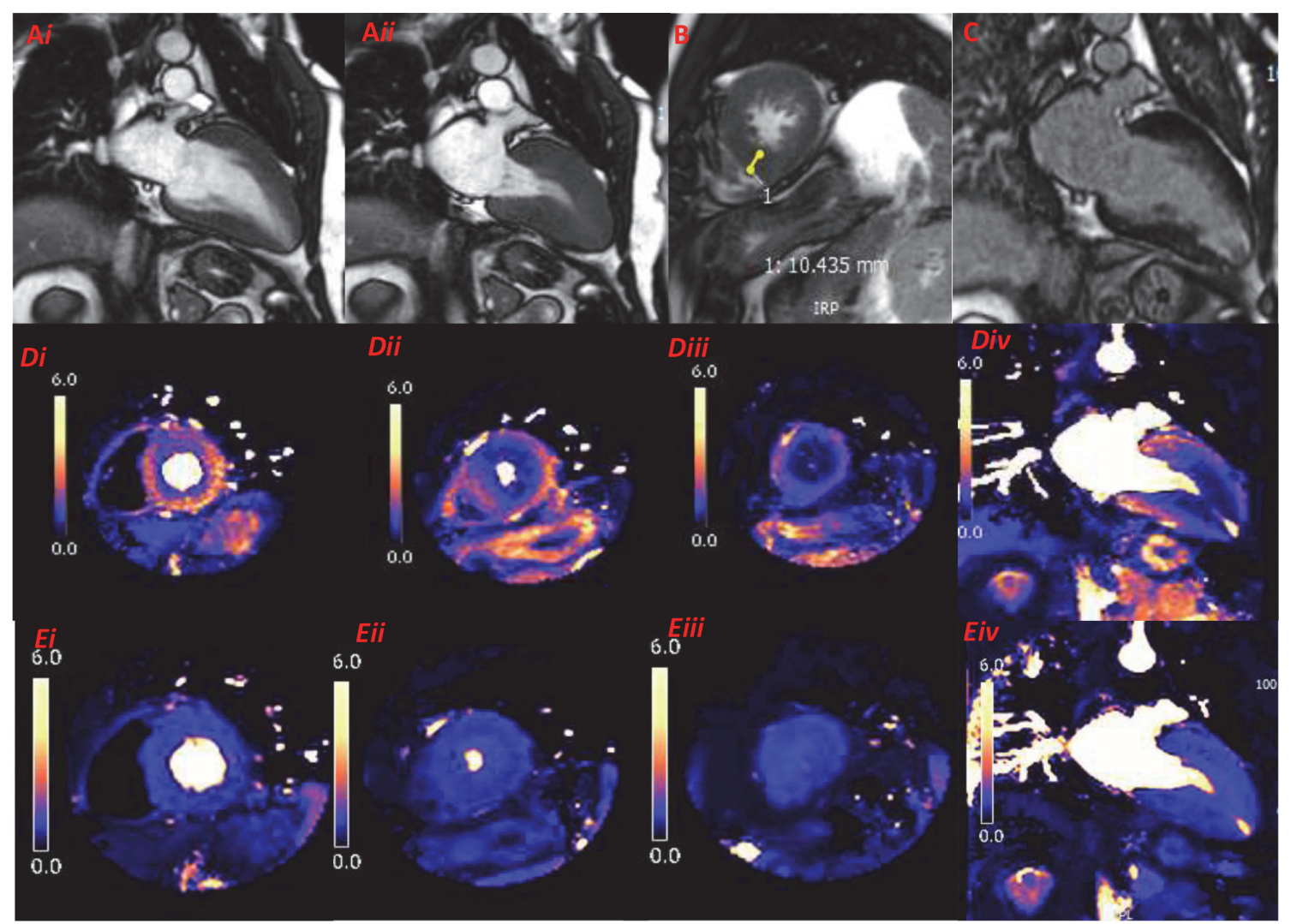

Abstract 20 Figure 1 Perfusion in relative apical hypertrophic cardiomyopathy with discrete apical chamber. 2-chamber cine in end-diastole(Ai) and end-systole(Aii). Short-axis view showing maximum wall thickness of $10.4 \mathrm{~mm}$ at the apex (B). Apical subendocardial late gadolinium enhancement (C). Perfusion mapping following adenosine-stress in the 3 short-axis slices and 2-chamber (Di-iv) demonstrate dense perfusion defect most notable in the apical subendocardium but extending to the basal anteroseptum. Rest perfusion images for comparison (Ei-iv).

from other HCM phenotypes (ECG, arrhythmogenicity, genotype, scar pattern, natural history). Clinically, apical hypoperfusion is frequently observed in ApHCM. We hypothesised that quantitative perfusion would be different and distinct in apical HCM.

Methods and Results Patients: 100 subjects with ApHCM, 50 with conventional HCM and 28 healthy volunteer controls. 33 of the ApHCM had 'relative ApHCM' - MWT $<15 \mathrm{~mm}$ but other key features of the disease - typical ECG, apex thicker than septum with loss of apical tapering and apical systolic cavity obliteration.

CMR: Quantitative perfusion mapping CMR using adenosine vasodilator stress. A visual read plus global and regional map segmentation was performed, with results expressed as myocardial blood flow (MBF, $\mathrm{ml} / \mathrm{g} / \mathrm{min}$ ) and myocardial perfusion reserve (MPR).

Results Of 100 ApHCM, all (100/100) had apical perfusion defects on visual read. There was also a high prevalence of perfusion defects in conventional HCM (45/50, 90\%, $\mathrm{p}=0.012)$, typically in hypertrophied areas. There were no defects in controls $(\mathrm{P}<0.005)$. Compared with HCM, global stress $\mathrm{MBF}$ in ApHCM was equivalent (mean (IQ range) 1.71 $\mathrm{ml} / \mathrm{g} / \mathrm{min}(1.48-2.06)$ vs $1.61 \mathrm{ml} / \mathrm{g} / \mathrm{min}(1.31-2.08), \mathrm{P}=0.436)$ and lower than controls $(2.59 \mathrm{ml} / \mathrm{g} / \mathrm{min}(2.28-2.96), \mathrm{P}<0.005$. The reduction in $\mathrm{MBF}$ was most pronounced in the apical segments in ApHCM vs HCM (1.27 $\mathrm{ml} / \mathrm{g} / \mathrm{min}(1.05-1.64)$ vs $1.59 \mathrm{ml} / \mathrm{g} / \mathrm{min}(1.25-2.06 \mathrm{ml} / \mathrm{g} / \mathrm{min}), \mathrm{P}<0.005)$ and controls $(2.54 \mathrm{ml} / \mathrm{g} / \mathrm{min}(2.34-3.35), \mathrm{P}<0.005)$. The defects were profound with flow reduction during stress being common $(\mathrm{MPR}<1)$. Flow reductions were less for relative ApHCM than overt ApHCM (global stress MBF $2.03 \mathrm{ml} / \mathrm{g} / \mathrm{min}$ (1.71$2.24)$ vs $1.62(1.36-1.89), \mathrm{P}<0.005)$, and similarly, relative ApHCM had less scar than overt ApHCM (FWHM LGE 0.0g $(0.0-7.0)$ vs $24.1 \mathrm{~g}(14.2-35.7), \mathrm{P}<0.005 ; 0.0 \%(0.0-7.6)$ vs 17.4\% (11.0-23.4\%), $\mathrm{P}<0.005)$.

Conclusions Apical microvascular ischaemia appears the hallmark feature of ApHCM, occurring even when the apical hypertrophy does not reach conventional diagnostic criteria (relative ApHCM).

\section{EXPLORING THE ETHNIC DIFFERENCES IN CARDIAC FUNCTION AND OUTCOMES: A UK BIOBANK STUDY}

1,2RS Motiwale, 'GP McCann, 'EM Brady. 'Department of Cardiovascular sciences, University of Leicester, NIHR Leicester Biomedical centre, Glenfield, Hospital, Leicester, LE3 9QP; ${ }^{2}$ School of Medicine, University of Leicester, LE1 7RH

10.1136/heartjnl-2021-BSCMR.21

Background Risk factors for heart failure (HF) are more prevalent among South Asians (SA). It is unclear whether this translates to a higher risk of HF. Some evidence suggests that there are baseline cardiovascular differences between SA and White Europeans (WE), however this has been previously based on echocardiography imaging which is less accurate compared to cardiac magnetic resonance (CMR).

Aims To investigate the differences in left ventricular (LV) volumes, cardiac output, CO, (both determined by CMR), incidence of $\mathrm{HF}$ and risk of $\mathrm{HF}$ between $\mathrm{SA}$ and WE, in a cohort without cardiovascular disease (CVD). 
Methods The core cohort $(502,655)$ was derived from the UK Biobank prospective study. Data from the CMR sub-study $(39,703)$ were used which utilised a 1.5 Tesla scanner and an automated scan analyser. Participants with a history of CVD and loop diuretic use were excluded. Baseline characteristics, including demographics, comorbidities, and biomarkers, were compared. LV volumes and CO were compared, and linearly regressed for an association with SA ethnicity. HF incidence rates were calculated from new HF hospitalisations (ICD10 codes: I50.0, I50.1, I50.9, I11.0, I13.0 and I13.2). Cox-proportional hazards analysis determined comparative risk of developing HF.

Results Ninety-four (1.3\%) of SA had HF and 4,218 (1.0\%) of WE had HF. Higher proportions of WE with HF had elevated levels of cholesterol and smoking history (e.g.: $41.2 \%$ vs. $11.7 \%$ of SA with $\mathrm{HF}, p<0.0001)$. Higher proportions of SA with HF had hypertension and type 2 diabetes $(33.0 \%$ vs. $9.0 \%, p<0.0001)$ and duration of diabetes (13 years vs. 7 years, $p=0.0018)$. All indexed LV parameters were smaller after adjustment for prevalent risk factors (e.g.: LV end diastolic volume reduced by $20.7 \mathrm{ml}, p<0.0001)$. A trend for a higher risk of HF was present in SA, which was attenuated by cholesterol (HR: 1.25, $p=0.072$ and HR: 1.11, $p=0.450$ ).

Conclusion A trend for a higher risk of HF is present in SA, potentially due to cholesterol and smaller cardiac structures promoting myocardial strain in maintaining ejection fraction. Future research should determine causes of new HF events and confirm subtypes.

\section{BSCMR annual meeting 2021 - abstract submission}

\section{INTER-FIELD STRENGTH AGREEMENT OF LEFT VENTRICULAR STRAIN AND STRAIN RATE USING TISSUE TRACKING AND AI DERIVED GLOBAL LONGITUDINAL SHORTENING}

${ }^{1}$ Sarah L Ayton, 1,2Gaurav S Gulsin, 'Kelly S Parke, 'Joanne V Wormleighton, 1J Ranjit Arnold, 'Alastair J Moss, ${ }^{1}$ Anvesha Singh, ${ }^{3}$ Hui Xue, ${ }^{3}$ Peter Kellman, ${ }^{1}$ Matthew PM Graham-Brown, 'Gerry P McCann. 'Department of Cardiovascular Sciences, University of Leicester and the NIHR Leicester Biomedical Research Centre, Glenfield Hospital, Leicester, UK; 'Department of Radiology, University of British Columbia, St Paul's Hospital, 1081 Burrard Street, Vancouver; ${ }^{3}$ National Heart, Lung and Blood Institute, National Institutes of Health, Bethesda, MD

\subsection{6/heartjnl-2021-BSCMR.22}

Background The aim of this study was to assess the inter-field strength agreement of left ventricular (LV) strain, strain rates and artificial intelligence (AI) landmark-based global longitudinal shortening at $1.5 \mathrm{~T}$ and $3 \mathrm{~T}$, using routinely acquired cardiovascular magnetic resonance (CMR) cine images.

Methods This was a prospective, randomised cross-over observational study in which healthy individuals completed two CMR scans at $1.5 \mathrm{~T}$ and $3 \mathrm{~T}$ in a randomised order within 30 minutes. Short and long-axis balanced steady state free precession cine imaging was acquired with retrospective electrocardiogram gating and scanning conditions standardised between field strengths. Analysis was undertaken offline by a single experienced observer blinded to participant details. CMR Tissue Tracking (cvi42, Circle Cardiovascular Imaging, Calgary,
Canada) was used to derive LV global longitudinal, circumferential and long and short axis radial systolic strain and peak systolic, early diastolic and late diastolic strain rates. Global longitudinal shortening was measured from long axis images using a fully automated method with an AI deep neural network model. All strain values are expressed as positive numbers.

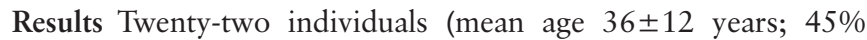
male) completed the study. There were no significant differences in blood pressure or heart rate during scanning between field strengths. Figure 1 shows scatterplots and Bland-Altman plots for LV systolic strain measurements and global longitudinal shortening at $1.5 \mathrm{~T}$ and $3 \mathrm{~T}$. Strain and strain rate
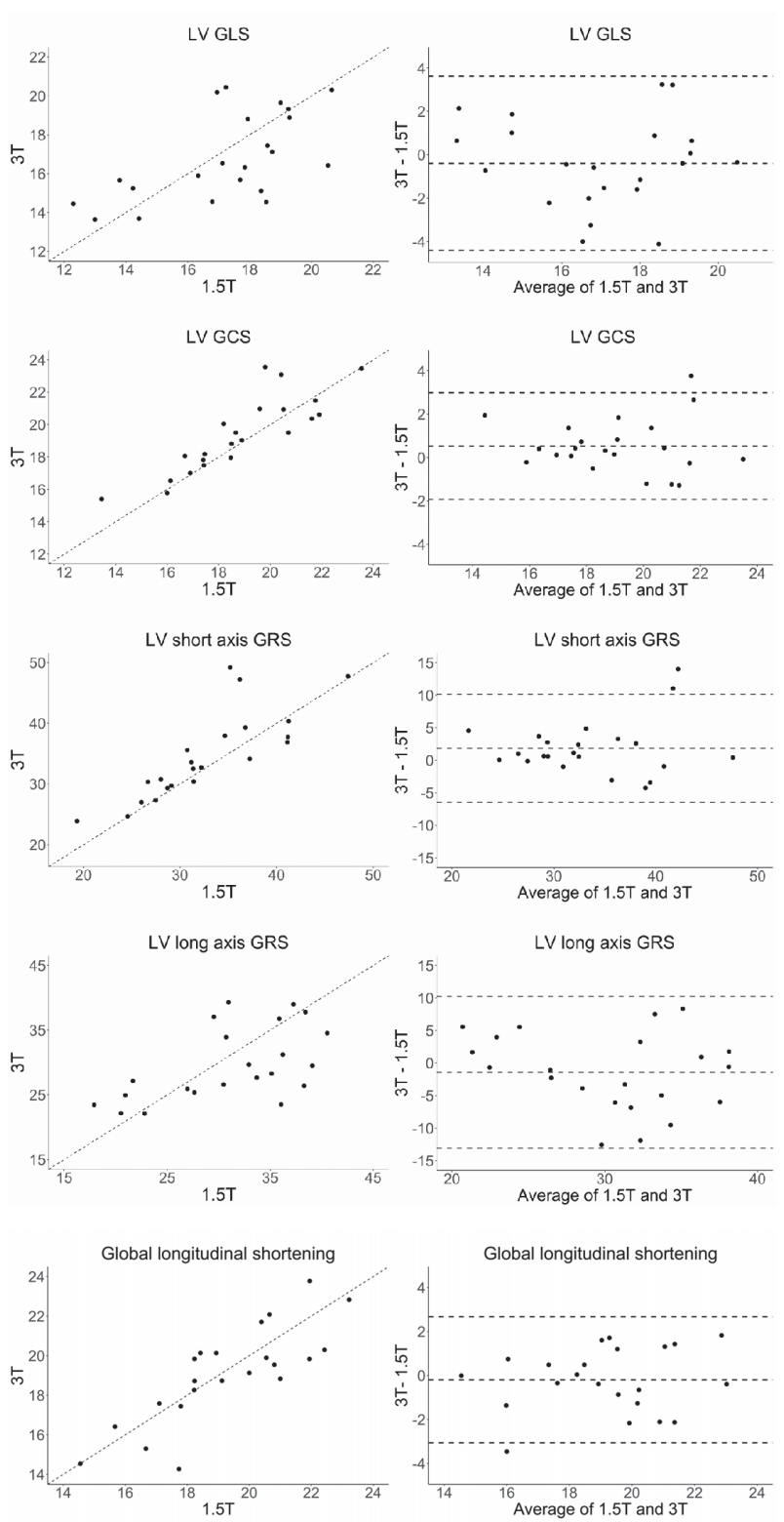

Abstract 22 Figure 1 Scatterplots (showing the unity line) and Bland-Altman plots for left ventricular global systolic strain and global longitudinal shortening measurements. GCS, global circumferential strain; GLS, global longitudinal strain; GRS, global radial strain; LV, left ventricular 